\title{
Spatial Distribution and Temporal Evolution of Scattering Centers by Optical Coherence Tomography in the Poly(L-Lactide) Backbone of a Bioresorbable Vascular Scaffold
}

\author{
Juan Luis Gutiérrez-Chico, MD, PhD; Maria D. Radu, MD; Roberto Diletti, MD; Alexander Sheehy, PhD;
} Mary Beth Kossuth, PhD; James P. Oberhauser, PhD; Thierry Glauser, PhD; Joel Harrington, PhD; Richard J. Rapoza, PhD; Yoshinobu Onuma, MD; Patrick W. Serruys, MD, PhD

\begin{abstract}
Background: Scattering centers (SC) are often observed with optical coherence tomography (OCT) in some struts of bioresorbable vascular scaffolds (BVS). These SC might be caused by crazes in the polymer during crimpdeployment (more frequent at inflection points) or by other processes, such as physiological loading or hydrolysis (eventually increasing with time). The spatial distribution and temporal evolution of SC in BVS might help to understand their meaning.
\end{abstract}

\begin{abstract}
Methods and Results: Three patients were randomly selected from 12 imaged with Fourier-domain OCT at both baseline and 6 months in the ABSORB cohort B study (NCT00856856). Frame-by-frame analysis of the SC distribution was performed using spread-out vessel charts, and the results from baseline and 6 months were compared. A total of 4,328 struts were analyzed. At baseline and follow-up all SC appeared at inflection points. No significant difference was observed between baseline and 6 months in the number of SC struts (14.9 vs. $14.5 \%, P=0.754)$ or in the distribution of SC. The proportion and distribution of SC did not vary substantially among the patients analyzed.
\end{abstract}

Conclusions: The SC observed in OCT imaging of the BVS are located exclusively at inflection points and do not increase with time. These findings strongly suggest that SC are caused by crazes in the polymer during crimp-deployment, ruling out any major role of hydrolysis or other time-dependent processes. (Circ J 2012; 76: 342-350)

Key Words: Coronary stenosis; Drug-eluting stents; Optical coherence; Poly(lactide); Tomography

$\mathbf{T}$ he Abbott Vascular bioresorbable vascular scaffold (BVS) (Santa Clara, CA, USA) consists of a semicrystalline poly(L-lactide) (PLLA) backbone and conformal coating of amorphous poly(D,L-lactide) (PDLLA) and the antiproliferative agent, everolimus. The molecular weight of the BVS polymers is degraded primarily through hydrolysis of the ester bonds present in each monomer subunit. Crystalline residues with a characteristic dimension less than $2 \mu \mathrm{m}$ are phagocytosed by macrophages. Ultimately, PLLA and PDLLA degrade to lactate, which is metabolised via the Krebs' cycle and other metabolic pathways, ${ }^{1}$ similarly to other biodegradable polymers. ${ }^{2}$ Complete polymer resorption occurs approximately 2 years after implantation. ${ }^{3,4}$ The BVS is laser-cut from a single piece of polymer tubing and then crimped onto a balloon. The structural design of the BVS consists of $19 \mathrm{~W}$-shaped rings connected longitudinally by straight links (Figure 1). The BVS has delivered acceptable and durable clinical and angiographic results up to 2 years post-procedure when the scaffold has been completely resorbed..$^{5,6}$

Unlike metallic stents, ${ }^{2,7-11}$ the translucency of the processed polylactide used in the Abbott Vascular BVS makes it particularly suitable for optical coherence tomography (OCT) imaging. The optical radiation can penetrate the translucent polymer, with significant backscattering occurring only at the borders of struts where the refractive index of the medium changes. Alternatively, the strut core has been characterized as a "black box", 3,5,6 signifying the absence of refractive index changes within the material (Figure 2). However, some OCT images show a focal hyperintense signal in the strut core without apparent contact with either the axial or transversal strut

Received July 19, 2011; revised manuscript received September 17, 2011; accepted October 7, 2011; released online November 19, 2011 Time for primary review: 38 days

Erasmus Medical Centre, Thoraxcentre, Interventional Cardiology Department, Rotterdam, The Netherlands (J.L.G.-C., M.D.R., R.D., Y.O., P.W.S.) and Abbott Vascular, Santa Clara, California, USA (A.S., M.B.K., J.P.O., T.G., J.H., R.J.R.)

Clinical trial registration: NCT00856856; URL: http://clinicaltrials.gov

Mailing address: Patrick W. Serruys, MD, PhD, FESC, FACC, Professor, Head of Interventional Cardiology, Erasmus MC, Thoraxcenter, Ba583a. 's-Gravendijkwal 230, 3015 CE Rotterdam, The Netherlands. E-mail: p.w.j.c.serruys@erasmusmc.nl

ISSN-1346-9843 doi:10.1253/circj.CJ-11-0726

All rights are reserved to the Japanese Circulation Society. For permissions, please e-mail: cj@j-circ.or.jp 


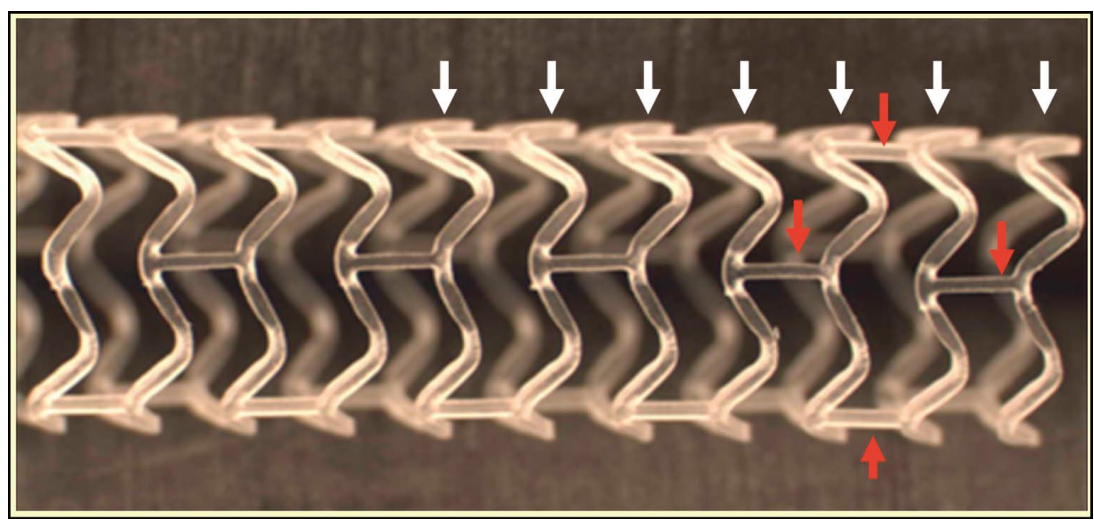

Figure 1. Structure of the Abbott Vascular bioresorbable vascular scaffold revision 1.1. An 18-mm length scaffold consists of $19 \mathrm{~W}$-shaped rings (white arrows), connected with the adjacent rings by 3 straight longitudinal units (red arrows).
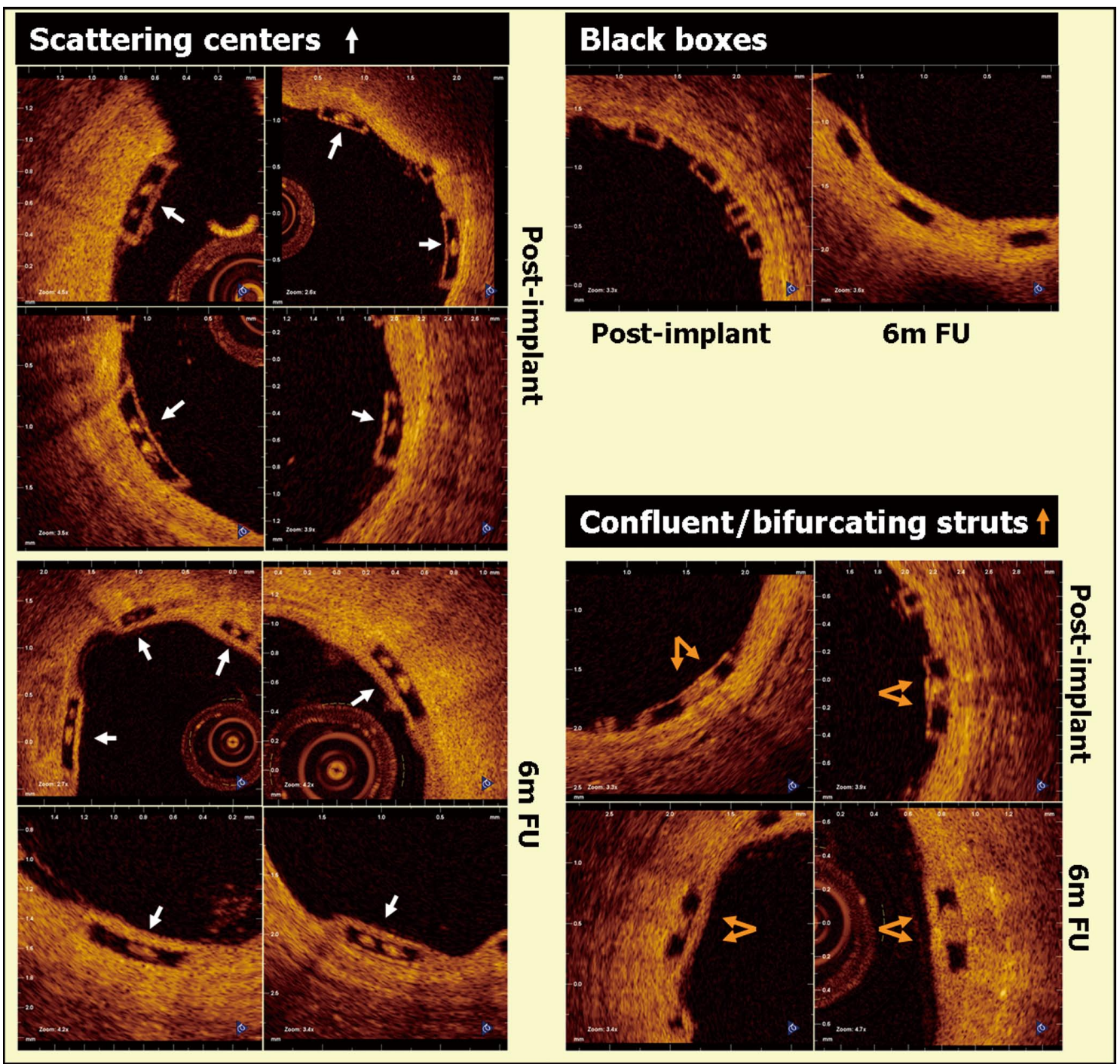

Figure 2. Definition of scattering centers (SC). The struts classified as having SC present a focal hyperintense backscattering signal in the core of the strut, which typically does not reach either the axial or the transversal strut edges, thus taking the appearance of a "dot" inside a box (Left). Notice the difference with the typical "black box" appearance of the BVS struts (Right upper). It is important to differentiate between SC from the optical appearance of confluent or bifurcating struts, where there is also a hyperintense signal in the core of the strut; however, in this case, it reaches its axial edges (Right lower). 


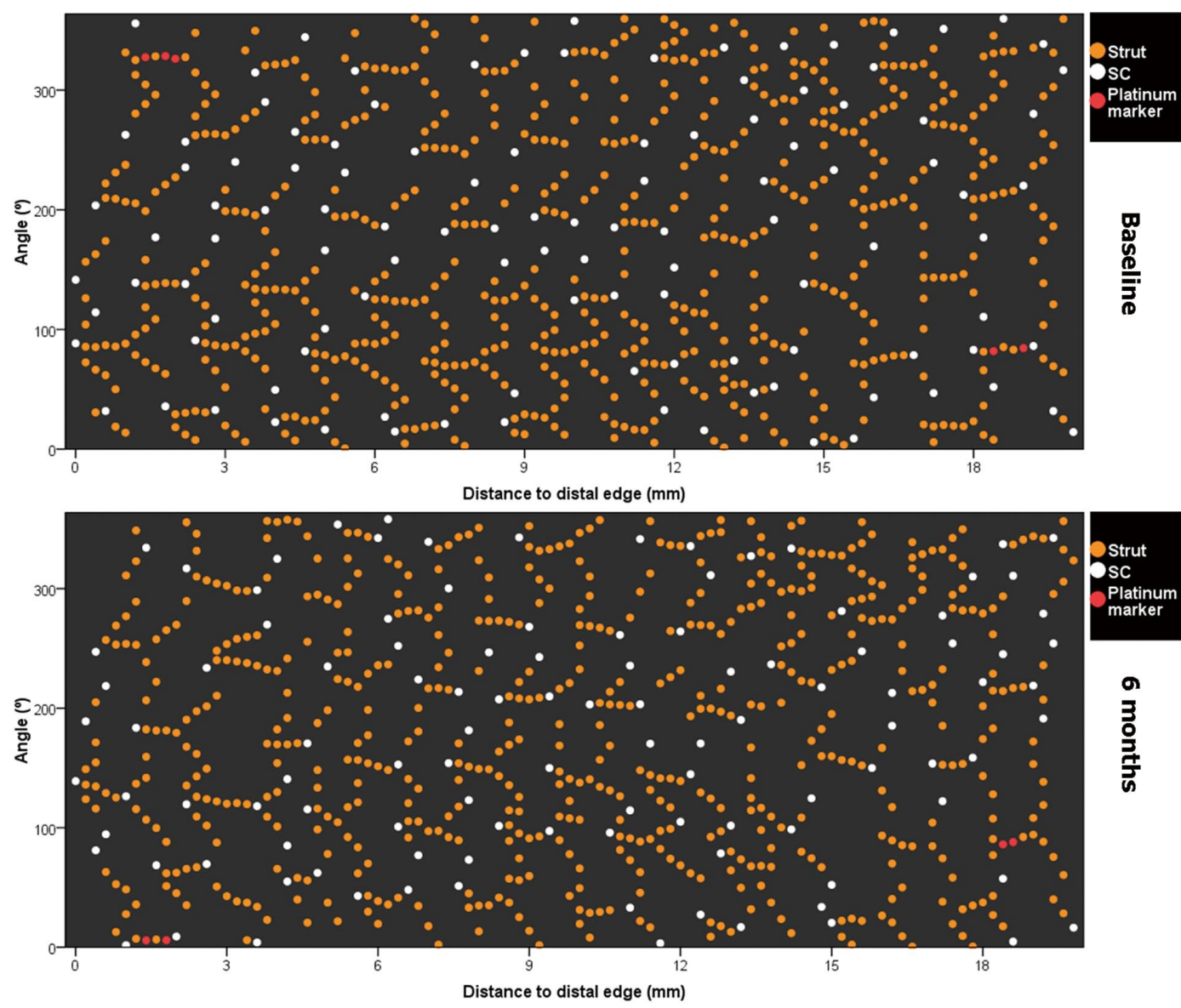

Figure 3. Patient 3: spread-out vessel chart showing the spatial distribution of the struts with scattering centers in the scaffolded vessel immediately post-implantation (baseline) and at the 6-month follow-up. The x-axis represents the distance from the distal edge of the stent to the strut; the $y$-axis represents the angle where the strut is located in the circular cross-section respect to the center of gravity of the vessel, taking as reference $0^{\circ}$ the position at $30^{\prime}$ clock. The result is a graphic representation of the spatial distribution of the struts along the scaffolded vessel as if it had been cut along the reference angle $\left(0^{\circ}\right)$ and spread out on a flat surface.

edges (Figure 2). The cause of this backscattering signal remains to be elucidated: it might reflect a change in the polymer's optical properties induced by the hydrolysis process or it might correspond to deformation-induced crazes in the backbone of the PLLA polymer, the latter preferentially located at hinge points of the BVS structure. The existence of regions with accelerated hydrolysis in humans or the presence of polymer crazes, eventually growing under continuous physiologic load, could both jeopardize the structural integrity of the BVS required to be functional.

This study explored the hypothesis that scattering centers (SC) observed in strut cores at baseline OCT imaging are derived from polymer crazing caused by the mechanical deformation of scaffold crimping and deployment, analyzing whether the spatial distribution of the SC at baseline is consistent with that hypothesis. The temporal evolution of these SC at 6 months permits assessment of the eventual influence of physiological loading, polymer degradation, or tissue integration in the genesis of the SC.

\section{Methods}

Study Sample

The ABSORB Cohort B registry (NCT00856856) design has been published elsewhere. ${ }^{3}$ The study enrolled patients older than 18 years, with diagnosis of stable or unstable angina pectoris or silent ischemia, and de novo lesions in native coronary arteries amenable for percutaneous treatment with the BVS (percent diameter stenosis $\geq 50 \%$ by visual estimation, reference vessel diameter $2.5-3.5 \mathrm{~mm}$ ). Exclusion criteria included: acute myocardial infarction, unstable arrhythmias, left ventricular ejection fraction $\leq 30 \%$, restenotic lesions, lesions located in the left main coronary artery or in bifurcations involving a side branch $>2 \mathrm{~mm}$, a second clinically or hemodynamically significant lesion in the target vessel, documentation of intracoronary thrombus, or initial TIMI 0 flow. For invasive follow-up purposes, the cohort was subdivided into 2 groups: cohort B1, undergoing multimodality invasive imaging (QCA, intravascular ultrasound, virtual histology, palpography, and OCT) at 6 and 24 months; and cohort B2, with an identical imaging follow-up protocol scheduled at 12 and 36 months. All of the study lesions were treated with the BVS Revision 1.1 design 


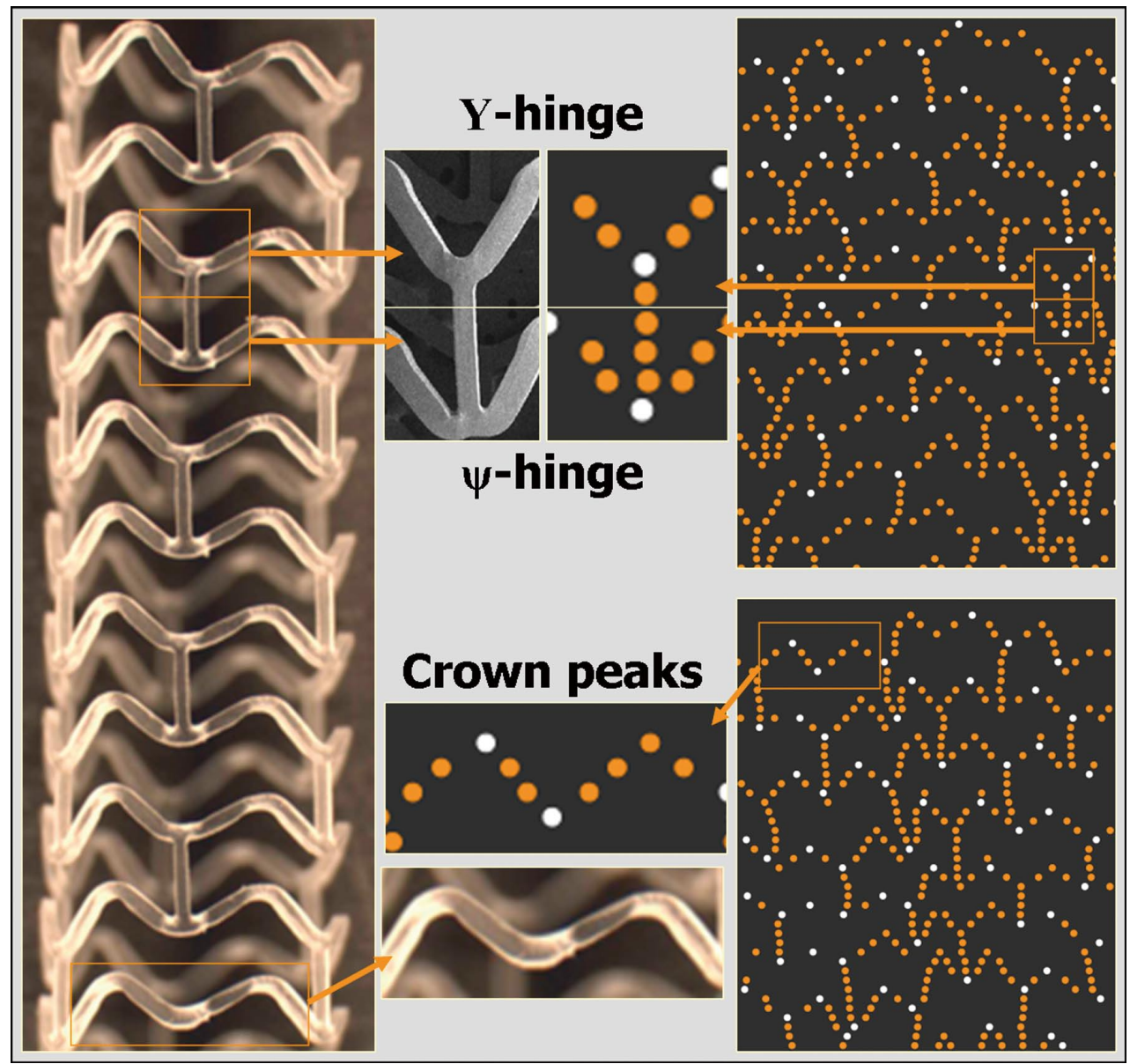

Figure 4. Schematic representation of the 3 types of hinges in the scaffold structure and their locations in the spread-out vessel diagram. The longitudinal connectors between the rings define inflection points that include $\psi$ - and Y-hinges (see schematic). Angulations in the zig-zag ring at a point where there is no longitudinal connector are denominated "crown peaks". The scaffold has 19 rings; each ring contains $3 \psi$-hinges, 3 Y-hinges and 6 crown peaks, except the most distal ring ( $3 \psi$-hinges and 9 crown peaks) and the most proximal ring ( 3 Y-hinges and 9 crown peaks).

$3.0 \times 18 \mathrm{~mm}$ (Figure 1). The registry was approved by the ethics committee at each participating institution, and each patient gave written informed consent before inclusion.

For the present pilot study, a random sample of 3 patients in cohort B1 was selected among those patients who had undergone OCT imaging with a Fourier-domain C7 system (Lightlab Imaging, Westford, MA, USA) at baseline and at the 6month follow-up.

\section{OCT Study}

OCT pullbacks were obtained at baseline and follow-up with a Fourier-domain C7 system using a Dragonfly catheter (Lightlab Imaging) at a rotation speed of 100 frames/s with a nonocclusive technique. ${ }^{12}$ After infusion of intracoronary nitroglycerine, the imaging wire was withdrawn by a motorized pullback at a constant speed of $20 \mathrm{~mm} / \mathrm{s}$, while Iodixanol 320 contrast (Visipaque ${ }^{\mathrm{TM}}$, GE Health Care, Cork, Ireland) was infused through the guiding catheter at a continuous rate of $2-6 \mathrm{ml} / \mathrm{min}$.

Two independent operators analyzed the selected OCT pullbacks offline using proprietary software (Lightlab Imaging). The region of interest was defined as that between the most distal and most proximal frames in which struts could be detected by OCT. In this region of interest, a frame-by-frame analysis (0.2-mm longitudinal intervals) was performed. In every cross-section the lumen contour was drawn and a marker set at the mid-point of the adluminal leading edge of each strut. The markers defined the circumferential position of the strut with respect to the center of gravity of the vessel as an angle, taking the position at 3 o'clock as the $0^{\circ}$ reference.

Struts were labeled as containing SC if one or more focal hyperintense regions could be seen in the core of the strut, separated from the axial and transverse strut border interfaces. Struts with hyperintense central regions extending to the lumen border were considered to be related to the optical appearance of bifurcating/confluent struts but not classified as SC. In some of these cases, an indentation between the 2 bifurcating/confluent struts could be identified (Figure 2). Two investigators classified the struts independently, and the discrepancies were resolved by consensus.

\section{Spread-Out Vessel Charts}

The spatial distribution of the struts along the scaffolded vessel was analyzed through spread-out vessel charts. These 


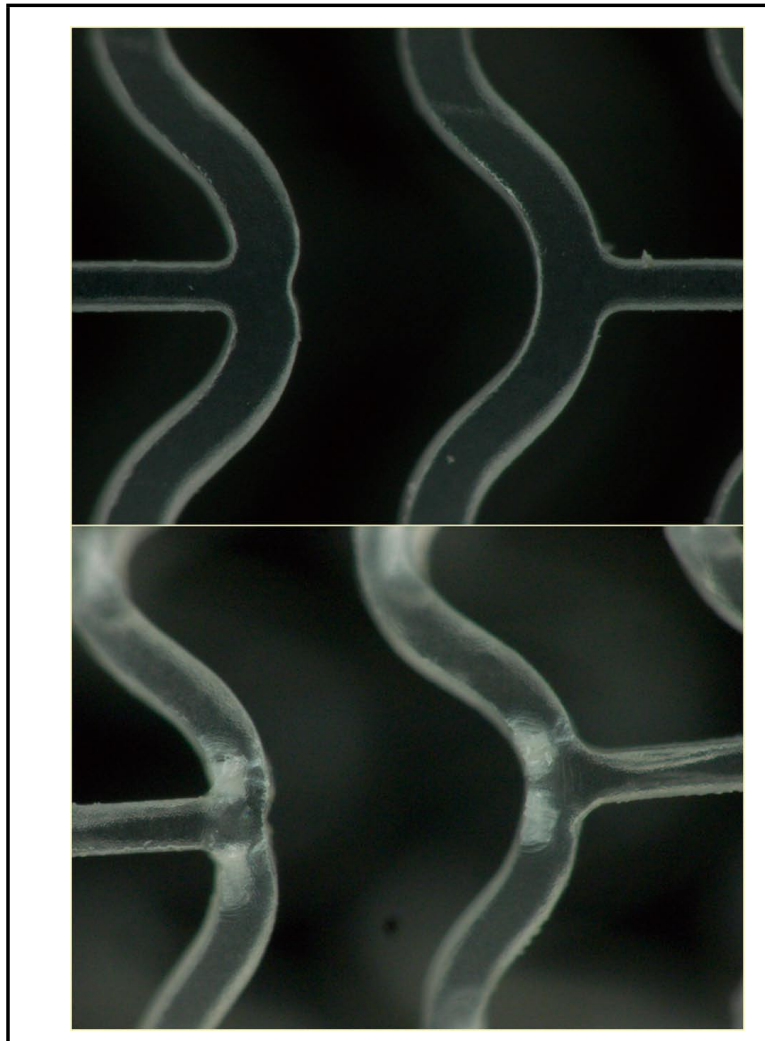

Figure 5. Representative images from the bench analysis of pre-crimp (Upper) and post-deployment (Lower), showing the stress-whitening at hinge points. Before crimp/deployment, scaffolds appeared transparent under optical microscopy. After deployment, scattering evidenced by increased opacity was observed at all hinge regions of the device.

graphics were obtained by correlating the longitudinal distance of each strut from the distal edge of the scaffold (abscises) with the angle defining its circumferential position with respect to the center of gravity of the vessel (ordinates) in each OCT pullback. ${ }^{13,14}$ The resulting charts represent the scaffolded vessel as if it had been cut longitudinally along the reference angle $0^{\circ}$ and spread out on a flat surface (Figure 3 ).

\section{Inflection Points (Hinges) Analysis}

The results displayed on the spread-out vessel charts permit visual identification of the different inflection points ${ }^{15}$ in the scaffold structure. The inflection points were classified into 3 types (Figure 4): (1) $\psi$-hinges (psi-hinges) at the distal part of a longitudinal connector, where the angle between the connector and the W-shaped ring is acute; (2) $\Upsilon$-hinges (ipsilonhinges) at the proximal part of a longitudinal connector, where the angle between the connector and the $\mathrm{W}$-shaped ring is obtuse; (3) crown peaks: hinges in the W-shaped rings at inflection points with no longitudinal connector. A $3.0 \times 18 \mathrm{~mm}$ BVS consists of $19 \mathrm{~W}$-shaped rings, 54 longitudinal connectors and 120 crown peaks. In each BVS ring $3 \psi$-hinges, $3 \Upsilon$-hinges, and 6 crown peaks can be identified, except in the most distal ( $3 \psi$-hinges and 9 crown peaks) and the most proximal ( $3 \Upsilon$ hinges and 9 crown peaks).

At least 4 points are required to define the shape of $\psi$ or $\Upsilon$, whereas 3 points are sufficient to define the shape of a crown peak. A hinge was considered associated with SC if SC appeared in any of the struts minimally required to define the hinge shape around the inflection point.

Individual tracking of the temporal evolution of each hinge was performed by matching the hinges in the spread-out vessel charts at baseline and 6-month follow-up.

\section{Bench Analysis}

Devices with the same design as the BVS in cohort B were used for a bench evaluation. Scaffolds were analyzed under light microscopy for the presence of enhanced opacity caused by scattering. Three uncrimped and 3 deployed devices were examined with optical microscopy (Keyence VHX-600) with incident light to maximize scattering within the bulk of the polymer and minimize surface reflection. SC were defined as areas of whitening greater than $20 \mu \mathrm{m}$ in diameter (Figure 5). The spatial distribution of SC along the scaffolded vessel was recorded using the spread-out vessel charts.

\section{Statistical Analysis}

The fraction of struts and hinge points with SC was calculated and the results compared with Pearson's chi-square test. The ability of OCT to track SC hinges between the baseline and follow-up pullbacks of the same patient was tested with a weighted $\kappa$ coefficient for the number count of white-dotted hinges per ring. Interobserver variability for the count of struts and of struts containing SC was calculated per cross-section by Kendall's tau-b and Spearman correlations. All the analyses and graphics were performed with the PASW 17.0.2 statistical package (SPSS Inc, Chicago, IL, USA).

\section{Results}

The 24 patients in the ABSORB cohort B1 underwent OCT at baseline and at 6-month follow-up; in 12 of these cases, the images were acquired with a Fourier-domain C7 system. No case was excluded from the analysis because of image quality issues. From these 12 cases, 3 were randomly selected for the spatial and temporal analysis of the SC. In all cases but one (patient 1, baseline OCT), the operator removed the guidewire before starting the OCT pullback to improve the image quality.

A total of 4,328 struts were analyzed: 1,502 in patient 1 (718 at baseline, 784 at 6 months), 1,403 in patient 2 (700 at baseline, 703 at 6 months) and 1,423 in patient 3 (728 at baseline, 695 at 6 months). The interobserver agreement was excellent for the count of struts per cross-section (Kendall's tau$\mathrm{b}=0.872$, Spearman rho $=0.932$ at baseline, $\mathrm{P}<0.001$; Kendall's tau-b=0.851, Spearman rho=0.912 at follow-up, $\mathrm{P}<0.001)$, and for the count of struts containing SC (Kendall's tau-b=0.936, Spearman rho $=0.959$ at baseline, $\mathrm{P}<0.001$; Kendall's tau$\mathrm{b}=0.954$, Spearman rho $=0.971$ at follow-up, $\mathrm{P}<0.001$ ). Analyst 1 tended to count on average 0.18 struts more per crosssection than analyst 2 at baseline $(\mathrm{P}=0.014)$, and 0.22 struts more at follow-up $(\mathrm{P}=0.04)$. No significant interobserver bias was detected in the count of struts with SC.

Analysis of the proportion of SC with respect to the total number of struts showed no significant difference between baseline and follow-up in any of the patients (Table 1). No significant difference was found between patients for the proportion of $\mathrm{SC}$ at any time point.

Figures 4, S1 and S2 present the spread-out vessel charts at baseline and 6-month follow-up of the 3 patients. All of the SC were located around hinges; no SC were detected in straight segments. Some hinges were sliced in different cross-sections because of the coincidence with systolic cardiac motion; in those cases it can be seen that the SC are located in the inner curvature of the bending (acute angle), without corresponding 


\begin{tabular}{|lccc|}
\hline $\begin{array}{l}\text { Table 1. Proportion of Struts With Scattering Centers Immediately Post-Implantation and at the 6-Month } \\
\text { Follow-up }\end{array}$ & Post-implant & 6 months & P value \\
Patient 1 & & & 0.919 \\
No. of struts & 718 & 784 & \\
SC & $91(12.7 \%)$ & $98(12.5 \%)$ & 0.586 \\
Patient 2 & & & \\
No. of struts & 700 & 703 & 0.818 \\
SC & $116(16.6 \%)$ & $109(15.5 \%)$ & \\
Patient 3 & 728 & 695 & 0.754 \\
No. of struts & $112(15.4 \%)$ & $110(15.8 \%)$ & \\
SC & & & \\
Total & 2,146 & 2,182 & \\
No. of struts & $319(14.9 \%)$ & $317(14.5 \%)$ & \\
SC & 0.106 & 0.130 & \\
Patient 1 vs. Patient 2 (P value) & & & \\
\hline
\end{tabular}

$\mathrm{SC}$, scattering centers. Data are $\mathrm{n}(\%)$

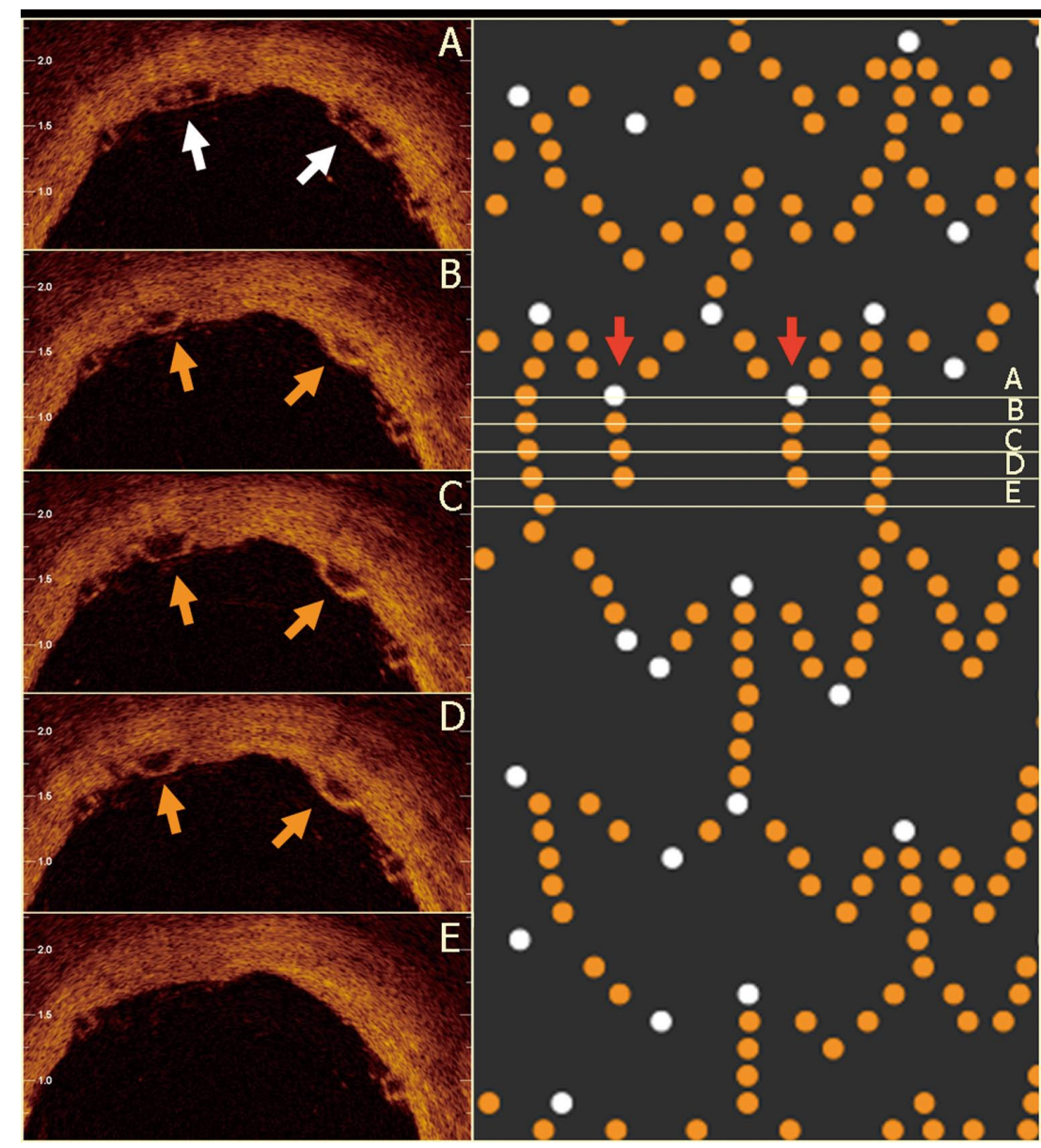

Figure 6. Hinges sliced in different cross-sections because of the coincidence with systolic cardiac motion (vessel and catheter moving in the same direction). The image shows 2 crown peaks with scattering centers and illustrates how the scattering is located in the inner curvature of the hinge (right side, red arrows; $\mathbf{A}$ ), with no scattering in the slices corresponding to the outer curvature (B-E).

$\mathrm{SC}$ in the outer curvature (obtuse angle) (Figure 6).

A comparison of the proportion of SC hinges did not show any significant difference between baseline and follow-up or between patients (Table 2).

Visual analysis of Figures 4-6 highlights the difficulty of tracking specific SC between baseline and the 6-month follow-up. The spatial distribution of the SC did not match exactly, suggesting that different features are revealed with any given pullback. To assess the ability to match the SC location at the 2 time points, the agreement in the number count of white-dotted hinges per ring between baseline and follow-up was tested with a weighted $\kappa$, which showed very poor agreement between the different hinges: best agreement for $\psi$ hinges (weighted $\kappa$ range $0.23-0.62$ ), for the rest of hinges, 


\begin{tabular}{|c|c|c|c|}
\hline & Post-implant & 6 months & $P$ value \\
\hline \multicolumn{4}{|l|}{ Patient $1^{*}$} \\
\hline$\psi$-hinges $(n=54)$ & $27(54.0 \%)$ & $33(61.1 \%)$ & 0.463 \\
\hline Y-hinges $(n=54)$ & $25(49.0 \%)$ & $27(50.0 \%)$ & 0.920 \\
\hline Crown peaks $(n=120)$ & $36(31.6 \%)$ & $37(30.8 \%)$ & 0.902 \\
\hline All inflection points $(n=228)$ & $88(40.9 \%)$ & $97(42.5 \%)$ & 0.731 \\
\hline \multicolumn{4}{|l|}{ Patient 2} \\
\hline$\psi$-hinges $(n=54)$ & $34(63.0 \%)$ & $36(66.7 \%)$ & 0.687 \\
\hline Y-hinges $(n=54)$ & $25(46.3 \%)$ & $25(46.3 \%)$ & 1.000 \\
\hline Crown peaks $(n=120)$ & $53(44.2 \%)$ & $45(37.5 \%)$ & 0.293 \\
\hline All hinges $(n=228)$ & $112(49.1 \%)$ & $106(46.5 \%)$ & 0.574 \\
\hline \multicolumn{4}{|l|}{ Patient 3} \\
\hline$\psi$-hinges $(n=54)$ & 38 (70.4\%) & $41(75.9 \%)$ & 0.515 \\
\hline Y-hinges $(n=54)$ & $21(38.9 \%)$ & $27(50.0 \%)$ & 0.245 \\
\hline Crown peaks $(n=120)$ & $53(43.3 \%)$ & $42(35.0 \%)$ & 0.186 \\
\hline All hinges $(n=228)$ & $111(48.7 \%)$ & $110(48.2 \%)$ & 0.925 \\
\hline \multicolumn{4}{|l|}{ Between patients ( $P$ value) } \\
\hline$\psi$-hinges & 0.226 & 0.250 & \\
\hline Y-hinges & 0.555 & 0.906 & \\
\hline Crown peaks & 0.091 & 0.547 & \\
\hline All hinges & 0.154 & 0.457 & \\
\hline
\end{tabular}

Data are $\mathrm{n}(\%)$

*In patient 1 only $50 \psi$-hinges, 51 Y-hinges and 114 crown peaks were analyzable at baseline, because of the presence of a guidewire in the pullback (Table presents corrected \%).

weighted $\kappa<0.34$ (Table S1).

In the bench study, a total of 684 hinge regions in each group of uncrimped and deployed devices were evaluated by optical microscopy. Analysis revealed the complete absence of SC in all regions of the uncrimped devices. In contrast, after deployment there were $\mathrm{SC}$ in all hinge regions (Figure 3).

\section{Discussion}

In this study we used a novel methodology for analyzing morphological changes in the polymeric scaffold of a BVS using OCT. Spread-out vessel charts have been previously described in trials comparing the performance of intracoronary devices using OCT per strut analysis..$^{13,14,16}$ They are simple and intuitive graphic representations of the spatial distribution and clustering of the studied phenomenon, in this case of the SC along the BVS, providing insight into how the scaffold material may be changing over time post-implantation. This study is the first to use spread-out vessel charts as the primary tool for detailed spatial analysis, and the results presented here prove its potential and motivate future development of the technique. This methodology may complement 3D-rendering technologies, which are appealing imaging tools but limited in analytical capability.

The spread-out vessel charts presented here also illustrate another unanticipated phenomenon, namely, the effect of cardiac motion on the images obtained during the OCT pullback. One can see the elongation of the segments imaged in systole (when the vessel moves in the same direction as the pullback catheter) and the compression of the segments imaged in diastole (when vessel and pullback move in opposite directions).

Visual analysis of the struts displayed in the spread-out vessel charts showed that all of the SC were located at hinge points. OCT relies upon changes in refractive index on a length scale greater than that of the wavelength of light used in the instrument to create signal intensity. ${ }^{17,18}$ Thus, the presence of $\mathrm{SC}$ within the strut core is suggestive of the creation of new interfaces in the polymeric material, leading to microscopic changes in the refractive index. In vivo, mechanical deformation, polymer molecular weight degradation, and tissue integration are all potential causes of refractive index changes. The exclusive location of the SC at hinge points strongly suggests that they represent micro-crazes in the polymer. Deformationinduced crazing has long been demonstrated in both amorphous and semicrystalline polymers. ${ }^{19}$ In semicrystalline polymers, crazes are typically microvoids traversed by fibrils in the direction of the applied stress, a combination that preserves the strength of the material while creating a discontinuity in the index of refraction. ${ }^{19-21}$ The BVS undergoes varying magnitudes of mechanical deformation during crimping and deployment, depending on the location on the scaffold, but the highest strain (and stress) is experienced at the hinge points, the points about which the scaffold collapses and expands during crimping and deployment, respectively. Optically, this deformation manifests in the deployed device as stress-whitening at the hinge points (Figure 3), which is also evidence of crazing. The spatial distribution of the SC in OCT parallels that of the crazing observed in optical microscopy, clustering around the hinge points and leaving the scaffold areas that experience little or no mechanical deformation as unblemished "black boxes".

Though hydrolysis is a natural part of PLLA degradation, the presence of SC immediately post-implantation in a proportion comparable to that at the 6-month follow-up, likely rules out the involvement of hydrolysis in the genesis of these images during the first 6 months. Likewise, the similar proportion of $\mathrm{SC}$ at baseline and at the 6-month follow-up suggests that other processes, such as physiological loading, induce minimal crazing of the polymer after deployment. Therefore, the structural integrity of the scaffold is not jeopardized by hydrolysis or 
crazing up to 6 months. The preservation of structural integrity, especially in the first 3 months after implantation (the so-called "revascularization phase") is of the utmost importance to counteract vessel recoil and adverse remodeling, hence maintaining the initial luminal gain. ${ }^{1}$ Our finding is consistent with the excellent clinical outcomes reported for the BVS to date.,3,6

Previous studies from our group unsuccessfully attempted to track the individual SC between baseline and follow-up using 3D-rendering technologies (unpublished data). The current approach offers a clearer and more visual representation of the spatial location of the SC, but still fails to track them individually between 2 time points. Furthermore, the poor agreement between baseline and follow-up regarding the SC count per ring discourages any further attempt of matching. At a rotation speed of $100 \mathrm{~Hz}$ and a pullback speed of $20 \mathrm{~mm} / \mathrm{s}$, any feature smaller than $200 \mu \mathrm{m}$ will be difficult to capture in multiple pullbacks, and the OCT study should be rather considered a random sampling of the study phenomenon. This might explain the failure to match individual SC, and also why OCT detected SC in only $43-49 \%$ of the hinges, even though light microscopy had shown stress-whitening in $100 \%$. Consistent with this, OCT had the highest sensitivity for SC detection in the largest hinges ( $\psi$-hinges: $61-76 \%$ ) and the lowest sensitivity in the smallest hinges (crown peaks: $31-44 \%$ ).

\section{Study Limitations}

The OCT longitudinal image co-registration is limited in this study because of the pullback and rotation speeds used in the acquisition, which are the standard for clinical applications with a Fourier-domain $\mathrm{C} 7$ system. This study is a retrospective analysis from patients included in a clinical study, aimed at interpreting intriguing imaging observations; therefore, the pullback speed could not be altered to examine the factors that may govern longitudinal co-registration. For in vitro, preclinical, or prospective designs, a slower pullback speed (eg, $10 \mathrm{~mm} / \mathrm{s}$ ) would improve the ability to reproducibly observe features smaller than $200 \mu \mathrm{m}$ and improve co-registration accuracy.

The presence of a guidewire in one of the pullbacks might affect the comparability of these OCT studies, because the projected shadow hides a random sector of the cross-section. We minimized this problem by analyzing the proportion of $\mathrm{SC}$ with respect to the number of visible struts/hinges in the pullback.

The similarity between anomalous SC and the images generated by confluent or bifurcating struts might have affected the results. Although the defined analysis methodology attempted to differentiate between these essentially different images and showed excellent reproducibility, classification imprecision cannot be totally excluded.

The compression of the images during diastole made the reconstruction of the BVS structure difficult in some segments. Although the reconstruction was possible in all cases, following the pattern of the device, the accuracy of the analysis is hampered in those segments.

\section{Conclusions}

The SC observed in the Abbott Vascular BVS are located exclusively at hinge points and present at baseline and 6-month follow-up in similar proportions, suggesting that these images may represent polymer crazing caused by mechanical deformation during the crimping and deployment processes, likely ruling out any role of hydrolysis in their genesis. The consistent number and location of the SC between baseline and the 6-month follow-up suggests that physiological loading is not inducing further crazing on length scales greater than the spatial resolution capability of OCT.

\section{Disclosures}

This study analyzes data from a registry sponsored by ABBOTT Vascular, Santa Clara, CA, USA. A. Sheehy, M.B. Kossuth, J.P. Oberhauser, T. Glauser, J. Harrington and R.J. Rapoza are employees of Abbott Vascular.

\section{References}

1. Oberhauser JP, Hossainy S, Rapoza RJ. Design principles and performance of bioresorbable polymeric vascular scaffolds. EuroIntervention Suppl 2009; 5(Suppl): F15-F22.

2. Kozuki A, Shite J, Shinke T, Miyoshi N, Sawada T, Hellig F, et al. STELLIUM 1: First-in-man follow-up evaluation of bioabsorbable polymer-coated paclitaxel-eluting stent. Circ J 2010; 74: 2089-2096.

3. Serruys PW, Onuma Y, Ormiston JA, De Bruyne B, Regar E, Dudek $\mathrm{D}$, et al. Evaluation of the second generation of a bioresorbable everolimus drug-eluting vascular scaffold for treatment of de novo coronary artery stenosis: 6-month clinical and imaging outcomes. Circulation 2010; 122: $2301-2312$.

4. Onuma Y, Serruys PW, Perkins LE, Okamura T, Gonzalo N, GarciaGarcia HM, et al. Intracoronary optical coherence tomography and histology at 1 month and 2, 3, and 4 years after implantation of everolimus-eluting bioresorbable vascular scaffolds in a porcine coronary artery model: An attempt to decipher the human optical coherence tomography images in the ABSORB Trial. Circulation 2010; 122: $2288-2300$.

5. Ormiston JA, Serruys PW, Regar E, Dudek D, Thuesen L, Webster MW, et al. A bioabsorbable everolimus-eluting coronary stent system for patients with single de-novo coronary artery lesions (ABSORB): A prospective open-label trial. Lancet 2008; 371: 899-907.

6. Serruys PW, Ormiston JA, Onuma Y, Regar E, Gonzalo N, GarciaGarcia HM, et al. A bioabsorbable everolimus-eluting coronary stent system (ABSORB): 2-year outcomes and results from multiple imaging methods. Lancet 2009; 373: 897-910.

7. Teramoto T, Ikeno F, Otake H, Lyons JK, van Beusekom HM, Fearon $\mathrm{WF}$, et al. Intriguing peri-strut low-intensity area detected by optical coherence tomography after coronary stent deployment. Circ J 2010; 74: $1257-1259$.

8. Kim JS, Kim JS, Kim TH, Fan C, Lee JM, Kim W, et al. Comparison of neointimal coverage of sirolimus-eluting stents and paclitaxel-eluting stents using optical coherence tomography at 9 months after implantation. Circ J 2010; 74: 320-326.

9. Miyoshi N, Shite J, Shinke T, Otake H, Tanino Y, Ogasawara D, et al. Comparison by optical coherence tomography of paclitaxel-eluting stents with sirolimus-eluting stents implanted in one coronary artery in one procedure: 6-month follow-up. Circ J 2010; 74: 903-908.

10. Liu Y, Imanishi T, Kubo T, Tanaka A, Kitabata H, Tanimoto T, et al. Assessment by optical coherence tomography of stent struts across side branch: Comparison of bare-metal stents and drug-elution stents. Circ J 2010; 75: 106-112.

11. Goto I, Itoh T, Kimura T, Fusazaki T, Matsui H, Sugawara S, et al. Morphological and quantitative analysis of vascular wall and neointimal hyperplasia after coronary stenting: Comparison of bare-metal and sirolimus-eluting stents using optical coherence tomography. Circ J 2011; 75: 1633-1640.

12. Prati F, Cera M, Ramazzotti V, Imola F, Giudice R, Giudice M, et al. From bench to bedside: A novel technique of acquiring OCT images. Circ J 2008; 72: 839-843.

13. Gutierrez-Chico JL, van Geuns RJ, Regar E, van der Giessen WJ, Kelbaek H, Saunamaki K, et al. Tissue coverage of a hydrophilic polymer-coated zotarolimus-eluting stent vs. a fluoropolymer-coated everolimus-eluting stent at 13-month follow-up: An optical coherence tomography substudy from the RESOLUTE All Comers trial. Eur Heart J 2011; 32: 2454-2463.

14. Gutiérrez-Chico J, van Geuns RJ, Koch K, Koolen J, Duckers HJ, Regar E, et al. Paclitaxel-coated balloon in combination with bare metal stent for treatment of de novo coronary lesions: An optical coherence tomography first-in-human randomized trial balloon-first vs. stent first. EuroIntervention 2011; 7: 711-722.

15. Sangiorgi G, Melzi G, Agostoni P, Cola C, Clementi F, Romitelli P, et al. Engineering aspects of stents design and their translation into clinical practice. Ann Ist Super Sanita 2007; 43: 89-100.

16. Gutiérrez-Chico J, Jüni P, García-García HM, Regar E, Nüesch E, Borgia F, et al. Long term tissue coverage of a biodegradable polylactide polymer-coated biolimus-eluting stent: Comparative sequential 
assessment with optical coherence tomography until complete resorption of the polymer. Am Heart J 2011 (in press).

17. Schmitt JM. Optical coherence tomography (OCT): A Review. IEEE J Sel Top Quantum Electron 1999; 5: 1205-1215.

18. Fujimoto J, Drexler W. Introduction to optical coherence tomography. In: Greenbaum E, editor. Biological and medical physics, biomedical engineering. Berlin: Springer, 2008; 1-45.

19. Olf HG, Peterlin A. Crazing and fracture in crystalline, isotactic polypropylene and the effect of morphology, gaseous environments, and temperature. J Polym Sci Polym Phys Ed 1974; 12: 2209-2251.

20. Kojima M. Stress whitening in crystalline propylene-ethylene block copolymers. J Macromol Sci B Physics 1981; 19: 523-541.

21. Liu Y, Kennard CHL, Truss RW, Calos NJ. Characterization of stresswhitening of tensile yielded isotactic polypropylene. Polymer 1997; 38: $2797-2805$.

\section{Supplemental Files}

Supplemental File 1

Figure S1. Patient 1: spread-out vessel chart showing the spatial distribution of the struts with scattering centers in the scaffolded vessel immediately post-implantation (baseline) and at the 6-month follow-up.

Figure S2. Patient 2: spread-out vessel chart showing the spatial distribution of the struts with scattering centers in the scaffolded vessel immediately post-implantation (baseline) and at the 6-month follow-up.

Table S1. Agreement on the Number of White-Dotted Hinge Points per Scaffold Ring Between Baseline and the 6-Month Follow-up

Please find supplemental file(s);

http://dx.doi.org/10.1253/circj.CJ-11-0726 